\title{
Oxygen saturation during endoscopic retrograde cholangiopancreatography: a comparison of two protocols of oxygen administration
}

\author{
J D Rigg, T C Watt, D E F Tweedle, D F Martin
}

\begin{abstract}
Patients having endoscopic retrograde cholangiopancreatography (ERCP) are generally elderly and require sedation while in the prone position. These factors may be expected to aggravate any risk of arterial hypoxia. This study evaluated two protocols of oxygen administration, one with and one without pre-oxygenation. In 25 patients in whom preoxygenation with 4 litres/minute for five minutes before sedation was used, followed by continuous oxygen administration, arterial oxygen saturation did not fall below $90 \%$ at any stage during the procedure. By contrast, in 25 patients who were not pre-oxygenated oxygen saturation fell below $90 \%$ in nine $(36 \%)$. As expected, hypoxia occurred most frequently during the early stages of sedation and endoscope insertion. Hypoxia did not occur in association with operations such as sphincterotomy, stone extraction or stent insertion. This study confirms that arterial hypoxia is a common event during ERCP and can be completely prevented by pre-oxygenation with four litres of oxygen given intranasally for five minutes before sedation.

(Gut 1994; 35: 408-411)
\end{abstract}

Patients having endoscopic retrograde cholangiopancreatography (ERCP) may be at particular risk of hypoxia for several reasons in addition to advanced age: (a) many have coincident cardiopulmonary disease; (b) sedation, often with a combination of benzodiazepine and an opioid, is necessary; (c) the procedure may be prolonged; (d) ERCP is routinely performed with the patient turned prone and sometimes in a darkened $x$ ray room.

In 1991 the British Society of Gastroenterology published their Recommendations for standards of sedation and patient monitoring during gastrointestinal endoscopy, ${ }^{1}$ based on the premise that minimising the risk of adverse cardiopulmonary events - especially arterial hypoxaemia would improve patient safety. This included the recommendation that supplemental oxygen should be given to 'at risk' patients (ASA grades III to $\mathrm{V})^{2}$ during endoscopy, and incorporated guidelines for the use of pulse oximetry and continuous electrocardiographic monitoring.

The Royal College of Surgeons ${ }^{3}$ has elaborated on these recommendations and proposes that all patients who receive sedation for any procedure, should receive supplemental oxygen and be monitored using pulse oximetry.

A detailed study of cardiorespiratory changes during complex endoscopic procedures ${ }^{+}$showed that arterial oxygen saturation decreased after sedation with benzodiazepine combined with an opiod and after introduction of the endoscope. Saturation remained below baseline throughout the examination and into the recovery period. Griffin et $a l,{ }^{5}$ in a study of 80 patients having ERCP, showed that $4.5 \mathrm{lpm}$ of intranasal oxygen started immediately before endoscopy prevented most, but not all, episodes of hypoxia. Oxygen saturation fell below $90 \%$ in 21 of 41 control patients and four of 39 patients given oxygen. Similarly in a recent study ${ }^{6}$ of elderly patients sedated with midazolam only for ERCP, three of 33 patients given intranasal oxygen at $2 \mathrm{lpm}$ required intervention for hypoxia. We performed this study to find out if the incidence of hypoxia during ERCP could be further reduced by pre-oxygenation before sedation, in addition to giving continuous intranasal oxygen during the procedure.

\section{Methods}

Fifty consecutive, unselected patients ( 25 male, 25 female, aged 24 to 88 years) having ERCP were studied. Age, weight, and ASA grade were recorded. All patients were given oxygen at $4 \mathrm{lpm}$ by nasal cannula (Argyle Ltd) and were alternately allocated into two groups:

Group 1 received oxygen after intravenous sedation and introduction of the endoscope into the duodenum.

Group 2 were pre-oxygenated for five minutes before intravenous sedation and continuously thereafter.

Oxygen at $4 \mathrm{lpm}$ was continued throughout

TABLE I ASA grade of patients having endoscopic retrograde cholangiopancreatography

\begin{tabular}{lcc}
\hline & \multicolumn{2}{c}{ No of patients } \\
\cline { 2 - 3 } ASA grade & Group 1 & Group 2 \\
\hline I (Normal, healthy) & 2 & 1 \\
II (Mild systemic disease) & 10 & 11 \\
III (Severe systemic disease) & 13 & 11 \\
IV (Life threatening disease) & 0 & 2 \\
V (Moribund) & 0 & 0 \\
\hline
\end{tabular}

TABLE II Doses of midazolam and nalbuphine

\begin{tabular}{lll}
\hline & Group 1 & Group 2 \\
\hline Midazolam & & \\
Range (mg) & $2 \cdot 5-15$ & $3-20$ \\
Mean (mg)(SD) & $7 \cdot 7(2 \cdot 9)$ & $8 \cdot 2(4 \cdot 3)$ \\
Mean (mg/kg)(SD) & $0 \cdot 123(0 \cdot 05)$ & $0 \cdot 125(0 \cdot 07)$ \\
Nalbuphine & & \\
Range (mg) & $0-10$ & $0-10$ \\
Mean (mg)(SD) & $5 \cdot 3(2 \cdot 5)$ & $5 \cdot 4(2 \cdot 3)$ \\
Mean (mg/kg)(SD) & $0 \cdot 0891(0.05)$ & $0 \cdot 082(0 \cdot 04)$ \\
\hline
\end{tabular}

South Manchester,

Manchester

Correspondence to:

Dr D F Martin, Department of

Radiology, Withington

M20 8LR.

Accepted for publication

14 July 1993 


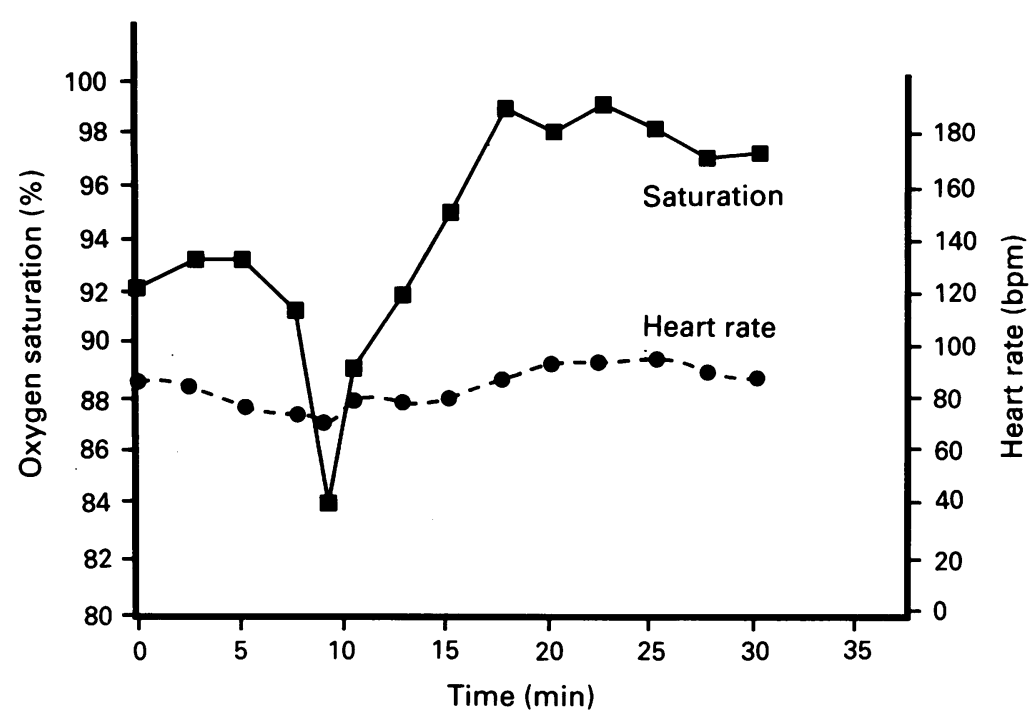

Figure 1: Typical recordings of oxygen saturation and pulse rate evaluations in a patient who did not receive pre-oxygenation.

the procedure in both groups, and in recovery until each patient was judged to be awake and alert.

Arterial oxygen saturation and heart rate were measured continuously using a Critikon Oxyshuttle pulse oximeter (Critikon Ltd, Berkshire) with the finger probe applied to the right hand, recordings being made at 2.5 minute intervals. Events such as insertion of the endoscope, change of position, drug administration and doses as well as operations (sphincterotomy, stone extraction, stenting) were noted. All measurements were made by independent observers (JDR or TCW).

Patients lay supine on the $x$ ray table at the start of the procedure to allow baseline measurements and application of nasal cannula, and were then turned to the left lateral position and the throat sprayed with lignocaine from a metered dose spray (7 puffs $=140 \mathrm{mg}$ ).

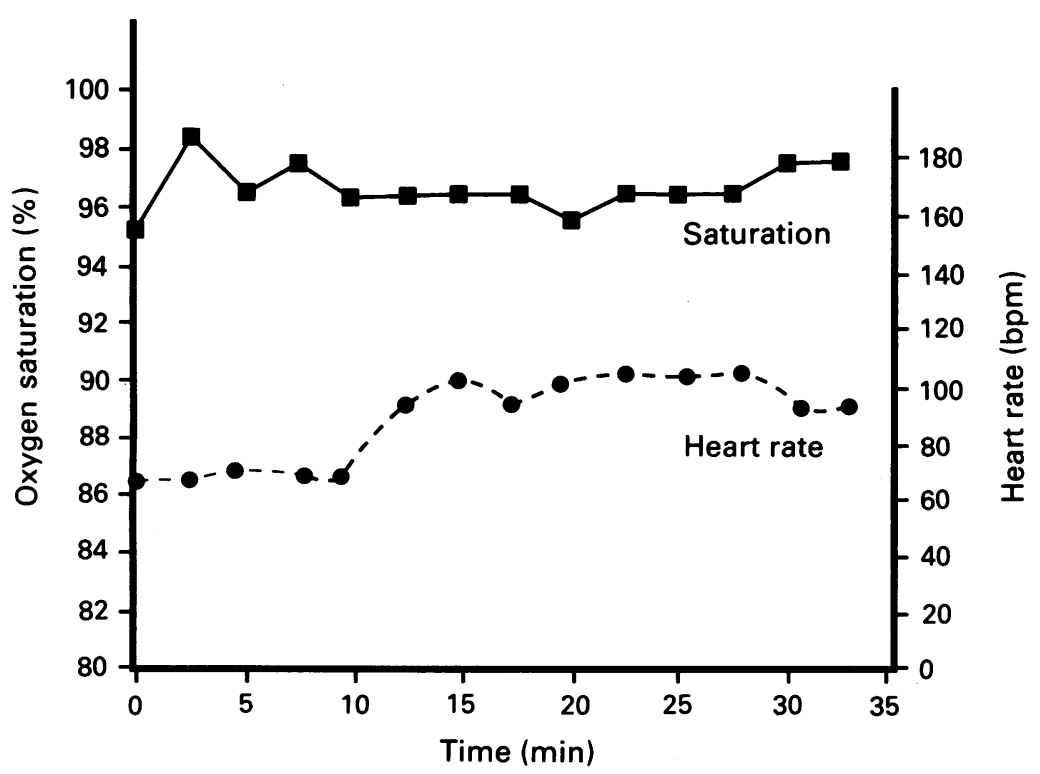

Figure 2: Typical recordings in a pre-oxygenated patient.
Nalbuphine (Nubain, Dupont Pharmaceuticals Ltd), followed by midazolam (Hypnovel, Roche Products Ltd) was given through an indwelling intravenous cannula until satisfactory sedation was achieved and the duodenoscope (Olympus JF1T10/TJF10) was then introduced. Patients were turned prone once the endoscope was positioned in the duodenum.

Boluses of intravenous hyoscine butylbromide $20 \mathrm{mg}$ (Buscopan, Boehringer-Ingelheim Ltd, Berkshire) or glucagon $0.5 \mathrm{mg}$ (Novo Nordisk Pharmaceuticals Ltd, West Sussex) were given as anti-spasmodics when required during the procedure and further incremental doses of sedation were given at the discretion of the endoscopist to maintain satisfactory patient comfort and compliance.

Approval for the study was obtained from the local ethical committee.

\section{Results}

The two groups were similar with respect to age (mean (SD) group $166 \cdot 1(17 \cdot 7)$ years, group 2 $63.2(16 \cdot 2)$, weight (group $162 \cdot 2(11 \cdot 4) \mathrm{kg}$, group $267 \cdot 4(12 \cdot 5) \mathrm{kg}$ ), and length of procedure (group 135 (15) min, group 237 (9) $\mathrm{min}$ ). Table I shows the ASA grade of patients in both groups. Table II shows the mean doses of sedative drugs, which were similar for the two groups.

Figures 1 and 2 illustrate typical patterns of oxygen saturation and heart rate during ERCP in group 1 (without pre-oxygenated) and group 2 (pre-oxygenated) patients respectively.

Table III shows the trends in oxygen saturation. Baseline saturation was similar in the two groups. After sedation and before turning the patient prone, median minimum saturation was $90 \%$ for group 1 and $96 \%$ for group 2 (MannWhitney $U$ test $p<0 \cdot 001)$. Minimum saturation after sedation was significantly lower than baseline in group 1 only. None of the pre-oxygenated group 2 subjects had oxygen saturations below $90 \%$ after sedation, compared with nine of 25 in group 1. After being turned prone all patients received intranasal oxygen and no patient's saturation fell below $90 \%$. No episodes of desaturation were noted in association with any operations.

Table IV shows the trends in heart rate. Mean heart rate at the time of lowest oxygen saturation was not significantly changed from baseline in either group. One patient in group 1 was noted to have an irregular pulse rate, 85 beats $/ \mathrm{min}$ at the time of maximal desaturation to $80 \%$; this resolved rapidly once the patient was turned prone and oxygen administration started. Twenty three of 25 patients in each group received at least one dose of intravenous hyoscine butylbromide $20 \mathrm{mg}$; four patients in group 1 and eight patients in group 2 received more than one dose. Mean heart rate was significantly increased in both groups after the first dose of hyoscine butylbromide.

\section{Discussion}

The value of pre-oxygenation and continuous oxygen administration during upper gastroin- 
TABLE III Trends in oxygen saturation during endoscopic retrograde cholangiopancreatography baseline $\mathrm{v}$ minimum saturation after sedation

\begin{tabular}{|c|c|c|c|c|}
\hline & \multicolumn{2}{|l|}{ Group 1} & \multicolumn{2}{|l|}{ Group 2} \\
\hline & Median & Range & Median & Range \\
\hline $\begin{array}{l}\text { Baseline saturation } \\
\text { Minimum saturation after sedation } \\
\text { Change in saturation. }\end{array}$ & $\begin{array}{r}95 \% \\
90 \% \\
-5 \%\end{array}$ & $\begin{array}{c}92-99 \% \\
80-98 \% \\
-16 \text { to }+1 \%\end{array}$ & $\begin{array}{r}95 \% \\
96 \% \\
0 \%\end{array}$ & $\begin{array}{l}94-97 \% \\
96-98 \%{ }^{\star} \\
-6 \text { to }+3 \%\end{array}$ \\
\hline
\end{tabular}

testinal endoscopy was shown by Bell et al. ${ }^{7}$ Arterial desaturation is common during ERCP when supplemental oxygen is not given. ${ }^{45} \mathrm{~A}$ variety of factors may contribute to this including the use of a large diameter endoscope, the pre-operative condition of the patient, and the need for sedation, which is usually greater than for diagnostic gastroscopy, often with both a benzodiazepine and an opioid; such combinations are associated with an increased risk of adverse cardiopulmonary events. ${ }^{38}$ In addition, patients may be partly concealed by $x$ ray equipment and placed in the prone position, which is associated with ventilatory impairment in spontaneously breathing patients. ${ }^{9}$

The recommendations for sedation and patient monitoring during gastrointestinal endoscopy ${ }^{1}$ include the provision of supplemental oxygen to ASA grades III to V patients. When endoscopy is being performed in a darkened room or with a large diameter endoscope or when combinations of benzodiazepines and opioids are used, the use of pulse oximetry to detect oxygen desaturation is recommended. Although the British Society of Gastroenterology recommendations formed the initial background to this study these are now superceded by the Royal College of Surgeons working party report, ${ }^{3}$ which suggests pre-oxygenation and continuous supplemental oxygen with oximetry for all sedated patients. The results of this study support those recommendations for patients having ERCP. It is significant that in this study and in previous studies ${ }^{4}$ oxygen saturation fell most dramatically at the start of the procedure - that is, after intravenous sedation and insertion of the endoscope. Griffin et $a l^{5}$ gave oxygen to half of their patients immediately before sedation and positioning of the endoscope and the incidence of hypoxia (oxygen saturation less than $\mathbf{9 0 \%}$ for $\mathbf{6 0}$ seconds or more) was approximately $10 \%-4$ of 39 patients, compared with 21 of 41 (approximately $50 \%$ ) controls who received no supplemental oxygen. Increasing age correlated with lower saturation in both groups and it is unacceptable to permit even $10 \%$ of elderly

TABLE IV Trends in heart rate $(H R)$ during endoscopic retrograde cholangiopancreatography

\begin{tabular}{|c|c|c|}
\hline & $\begin{array}{l}\text { Group I } \\
H R(b p m)(S D)\end{array}$ & $\begin{array}{l}\text { Group } 2 \\
H R(b p m)(S D)\end{array}$ \\
\hline \multirow{3}{*}{$\begin{array}{l}\text { Baseline } \\
\text { At minimum } \mathrm{SpO}_{2} \\
\text { Before iv hyoscine } \\
\text { butylbromide } 20 \mathrm{mg} \\
\text { After iv hyoscine } \\
\text { butylbromide } 20 \mathrm{mg}\end{array}$} & $\begin{array}{l}85.5(13.8) \\
82.6(15 \cdot 3)\end{array}$ & $\begin{array}{l}84 \cdot 6(16 \cdot 9) \\
83 \cdot 2(13 \cdot 3)\end{array}$ \\
\hline & $86 \cdot 2(12 \cdot 6)^{\star}$ & $80 \cdot 5(13 \cdot 3)^{\star \star}$ \\
\hline & $106 \cdot 2(18 \cdot 8)^{\star}$ & $98 \cdot 5(17 \cdot 2)^{\star \star}$ \\
\hline
\end{tabular}

${ }^{\star} \mathrm{p}<0.001,{ }^{\star \star} \mathrm{p}<0.001$ (Student's $t$ test)

iv =Intravenous; $\mathrm{SpO}_{2}=$ pulse oximetry. patients, who will probably have coincident cardiorespiratory problems, to become hypoxic during ERCP. The patients in Griffin's study who received oxygen would have had a variable period of pre-oxygenation before sedation. In this study we have compared oxygen saturation in patients pre-oxygenated for five minutes before sedation with patients given oxygen after sedation and endoscope insertion. Oxygen saturation fell significantly in group 1 , with nine of 25 patients having saturations below $90 \%$. In the pre-oxygenated group all patients maintained saturations of $90 \%$ or greater. After the start of intranasal oxygen, no patient in either group had a saturation below $90 \%$ and no episode of desaturation occured in association with any operation. We chose to use $4 \mathrm{lpm}$ of oxygen as this is the maximum volume suggested by the British Society of Gastroenterology ${ }^{1}$ and now by Royal College of Surgeons ${ }^{3}$ and is thought not to significantly put at risk of hypercapnia patients with chronic respiratory problems. Volumes less than $4 \mathrm{lpm}$ may not be sufficient to avoid hypoxia; Haines et $a l^{6}$ had a $10 \%$ need for intervention for hypoxia in ERCP patients sedated with midazolam alone who received preoxygenation with $2 \mathrm{lpm}$. In our study oxygen given at $4 \mathrm{lpm}$ was tolerated without difficulty by all patients.

Various techniques of pre-oxygenation have been studied ${ }^{111}$ and it has been shown that during apnoea, oxygenation is maintained for longer after a lengthy (two to four minutes) period of pre-oxygenation with $100 \%$ oxygen than after three or four vital capacity breaths. This may reflect greater 'washout' of nitrogen from the lungs and build up of oxygen stores. Similarly here, after a five minute pre-oxygenation period with $4 \mathrm{lpm}$ oxygen intranasally, oxygenation was well maintained even with sedation likely to cause respiratory depression.

Previous studies ${ }^{4}$ have reported tachycardia in association with arterial desaturation. We found no significant increase in heart rate at minimum saturation, although one patient was noted to have an irregular pulse when her saturation fell to $80 \%$. The lack of tachycardia may reflect the fact that desaturation was short lived with all patients eventually receiving supplemental oxygen for the greater part of the procedure. The single factor most obviously associated with an increase in heart rate was the intravenous injection of hyoscine butylbromide; this is a predictable effect of an anti-cholinergic that is in widespread use during upper gastrointestinal endoscopy. These findings are similar to those of Haines et $a l^{6}$ who reported no difference in the incidence of tachycardia (23\%) in patients having ERCP with or without supplemental oxygen who received $40 \mathrm{mg}$ hyoscine butylbromide routinely.

The results of this study support the Royal College of Surgeons recommendations that all patients having ERCP should receive prophylactic supplemental oxygen. Whether or not pulse oximetry is of value when supplemental oxygen administration removes the risk of hypoxia is debatable. Oximetry permits monitoring of pulse rate in addition to saturation and this may give reassurance to the endoscopist 
and assistants. More reliable devices are needed to avoid the frequent false alarms, which must detract from the potential benefit of monitoring with a pulse oximeter. A five minute period of pre-oxygenation at $4 \mathrm{lpm}$ by nasal cannula before sedation and continued throughout the endoscopy is an effective regimen for the prevention of hypoxaemia during ERCP.

1 Bell GD, McCloy RF, Charlton JE, Campbell D, Dent NA, Gear MWL, et al. Recommendations for standards of Gear MWL, et al. Recommendations for standards of sedation and patient monitoring
endoscopy. Gut 1991; 32: 823-7.

2 American Society of Anesthesiologists. New classification of physical status. Anesthesiology 1963; $24: 111$.

3 The Royal College of Surgeons of England Commission on the Provision of Surgical Services. Report of the working party on guidelines for sedation by non-anaesthetists, 1993 .

4 Murray AW, Morran CG, Kenny GNC, Macfarlane P, Anderson JR. Examination of cardiorespiratory changes during upper gastrointestinal endoscopy. Comparison of monitoring of arterial oxygen saturation, arterial pressure and the electrocardiogram. Anaesthesia $1991 ; 46: 181-4$.

5 Griffin SM, Chung SCS, Leung JWC, Li AKC. Effect of intranasal oxygen on hypoxia and tachycardia during endoscopic cholangiopancreatography. BMF 1990; 300: 83-4.

6 Haines DJ, Bibbey D, Green JRB. Does nasal oxygen reduce the cardio respiratory problems experienced by elderly patients undergoing endoscopic retrograde cholangiopancreatography. Gut 1992; 33: 973-5.

7 Bell GD, Bown S, Morden A, Coady T, Logan RFA. Prevention of hypoxaemia during upper gastrointestinal endoscopy by means of oxygen via nasal cannulae. Lancet 1987; i: $1022-4$.

8 Daneshmend TK, Bell GD, Logan RFA. Sedation for upper gastrointestinal endoscopy: results of a nationwide survey. Gut 1991; 32: 12-5.

9 Anderton JM, Keen RI, Neave R. The prone position. Anderton JM, Keen RI, Neave R, eds. London: Positioning the surgical patient. Butterworths, 1988: 43-61.

10 Valentine SJ, Marjot R, Monk CR. Pre-oxygenation in the elderly: a comparison of the four-maximal breath and three minute techniques. Anesth Analg 1990; 71: 516-9.

11 McCarthy E, Elliott P, Mirakhur RK, McLaughlin C. A comparison of different pre-oxygenation techniques in the elderly. Anaesthesia 1991; 46: 824-7. 